Voix et Images

voixetimages

\title{
Adrien Thério : critique littéraire, animateur polémiste et romancier. Entretien
}

\section{Donald Smith}

Volume 7, numéro 1, automne 1981

Adrien Thério

URI : https://id.erudit.org/iderudit/200300ar

DOI : https://doi.org/10.7202/200300ar

Aller au sommaire du numéro

Éditeur(s)

Les Presses de l'Université du Québec

\section{ISSN}

0318-9201 (imprimé)

1705-933X (numérique)

Découvrir la revue

\section{Citer ce document}

Smith, D. (1981). Adrien Thério : critique littéraire, animateur polémiste et romancier. Entretien. Voix et Images, 7(1), 7-26.

https://doi.org/10.7202/200300ar d'utilisation que vous pouvez consulter en ligne.

https://apropos.erudit.org/fr/usagers/politique-dutilisation/ 


\title{
Adrien Thério: critique littéraire, animateur polémiste et romancier
}

\author{
Entretien avec Donald Smith
}

Il était grand temps que l'on prépare un spécial Adrien Thério. Je suis convaincu que Thério, par ses multiples activités comme écrivain, animateur, organisateur et professeur, est une des personnalités les plus importantes de la littérature québécoise. Sans Thério, le Québec n'aurait jamais eu ces revues essentielles que sont Livres et Auteurs québécois et Lettres québécoises. Sans Thério, le journaliste Jules Fournier aurait sans doute été moins connu du public, les qualités d'écrivain d'Ignace Bourget, deuxième évêque de Montréal, auraient peut-être été passées sous silence. Sans Thério, d'innombrables professeurs et étudiants auraient moins eu le goût, la passion de la littérature d'ici. Et sans lui, le Québec aurait été privé d'un écrivain talentueux, prolifique, souvent fascinant. Je n'hésite pas à parler du "phénomène Thério», car c'est toute une institution littéraire qu'il a léguée au Québec. Et l'œuvre est loin d'être terminée.

Pour Adrien Thério, la littérature n'est pas un texte qu'on décortique froidement. Ce n'est pas une page morte qu'on passe à l'ordinateur, un contenu qu'on transforme en algorithmes. La littérature est vivante. Elle est l'expression profonde de soi. Elle est histoire, peinture, musique; elle est imagination transmise de génération en génération.

Afin de rendre justice à l'œuvre immense d'Adrien Thério, nous avons cru bon de diviser notre entrevue en trois parties: questions générales d'ordre biographique et bibliographique; questions sur Thério, critique littéraire. animateur et polémiste; commentaires et discussion sur Adrien Thério, écrivain. 
D.S. Vous êtes né à Saint-Modeste, comté de Rivière-du-Loup, qui deviendra, dans votre œuvre, le pays du Chemin Taché. C'est une région à laquelle vous êtes très attaché.

A.T. Je suis né à Saint-Modeste, à côté de Rivière-du-Loup, mais je n'y ai pas été élevé. J'avais cinq ans quand ma famille a déménagé au Chemin Taché, route rurale qui fait partie de Saint-Cyprien, situé à environ trente milles de Rivière-du-Loup, dans les terres. Ce n'est donc pas SaintModeste qui est devenu le Chemin Taché puisque le Chemin Taché existe. Je n'ai jamais parlé de Saint-Cyprien parce que c'est un nom que je n'aime pas. Mais Saint-Cyprien ne fait qu'un avec le Chemin Taché. Dans mon dernier récit, Saint-Cyprien devient Saint-Amable. C'est au fil des années que je me suis rendu compte jusqu'à quel point ce coin de terre ingrate m'avait marqué.

D.S. Les personnages du père et de la mère jouent un rôle important dans vos romans. II y a sûrement quelque chose d'autobiographique dans tout cela. Parlez-nous un peu de votre père et de votre mère.

A.T. J'ai été élevé dans une famille de type patriarcal. L'autorité, dans la maison, appartenait au père. J'en ai parfois souffert. II est sûr que ma mère était plus proche de ses enfants que mon père. La compréhension venait surtout de ce côté-là. Mon père n'osait pas vraiment manifester ses sentiments d'affection. Il fallait les deviner.

D.S. Est-ce que ce sont vos parents qui vous ont encouragé à aller à l'école, qui vous ont donné le goût de l'étude?

A.T. Ma mère sans doute parce qu'elle avait été institutrice quelques mois avant de se marier. Le métier ne lui avait pas plu. Elle devait avoir fait une sixième ou une septième année. Ce n'était pas si mal pour le temps. Je me souviens, en tout cas, qu'elle avait beaucoup d'idées à nous donner pendant que nous faisions nos devoirs après le souper, sur la grande table, dans la cuisine. Elle n'avait pas oublié ses règles de grammaire. Quant à mon père, comment aurait-il pu me donner le goût de l'étude. II ne savait ni lire ni écrire, contrairement à ses frères et sœurs. II tenait cependant à ce que nous allions à l'école. Je crois qu'il a regretté de ne pas y avoir été.

Mes frères aînés ont abandonné l'école en troisième année. J'ai répété ma quatrième parce que l'institutrice ne pouvait enseigner la cinquième. L'année suivante, une nouvelle institutrice $\mathrm{m}$ 'a fait faire ma cinquième, puis ma sixième et ma septième. Mais j'ai été le premier au Chemin Taché à suivre les cours de cinquième, sixième et septième années. Et j'étais seul dans ma classe. 
Il y a une autre personne, dans ma famille, qui m'a donné le goût de l'étude. C'est la sœur cadette de mon père dont le diplôme lui permettait d'enseigner à l'École Modèle de Saint-Épiphane. Elle enseignait à des élèves de dixième et onzième années. II n'y avait pas beaucoup de villages qui avaient le privilège d'offrir la dixième et la onzième, à ce moment-là. J'aimais beaucoup cette tante qui me le rendait bien d'ailleurs. Je crois qu'inconsciemment, c'est elle qui m'a donné le goût de m'instruire.

D.S. Lorsque vous étiez étudiant à l'Université Laval, vous avez été très malade. Il paraît que votre convalescence a joué un rôle décisif dans votre décision de devenir écrivain.

A.T. Ce n'est pas à l'Université Laval que je suis tombé malade pour la première fois. C'est au Séminaire de Rimouski, en philosophie première année. C'est en octobre 1945, je crois, que je suis parti pour le sanatorium de Mont-Joli où je suis resté trois ans. On y traitait les patients comme des chiens. Un jour, après une querelle avec le médecin-chef, je suis parti, de moi-même, pour le sanatorium de Sherbrooke où j'ai encore passé une année. À Sherbrooke, on traitait les patients comme des êtres humains. Je suis entré au sanatorium à l'âge de 19 ans, quand j'en suis sorti j'avais presque 24 ans. C'a été très difficile parce que je n'ai jamais été gravement malade. On m'annonçait ma sortie et à la dernière minute on me disait «non, il faut rester encore six mois». Les espoirs qu'on brise, ça finit par tuer. Je n'ai jamais parlé de cette période de ma vie probablement parce que le séjour à Mont-Joli me laisse trop de mauvais souvenirs. Mais c'est vrai que j'ai beaucoup lu pendant ces années et que j'ai voulu écrire une sorte de Menaud, texte que j'ai détruit. C'est pendant mon séjour à Mont-Joli que j'ai écrit mes premiers textes publiés: un conte dans XXième siècle, revue littéraire publiée à Ottawa, et un sketch dramatique joué à Radio-Canada en 1947, je crois, sous le titre d'Espoir accroché à la nuit. C'est ce sketch qui m'a fait connaître Yves Thériault. II m'a écrit à cette occasion. Nous avons découvert que nous étions parents.

En 1952, juste après avoir remis ma thèse de doctorat à la Faculté, j'ai fait une hémorragie soudaine. On m'a transporté d'urgence à l'Hôpital Laval. Je croyais que c'était de nouveau la tuberculose. Après quelques semaines d'examens, on s'est rendu compte que c'était une veine intérieure qui avait éclaté - surmenage m'a-t-on dit - et on m'a fait comprendre qu'il faudrait que je me tienne tranquille quelques mois. Cela m'a fait perdre un an. C'est pendant cette année de repos que j'ai obtenu une bourse de la Rockefeller Foundation pour aller étudier la littérature américaine à Harvard. J'y suis allé l'année suivante. Ce qui fait que, finalement, j'ai défendu ma thèse de doctorat alors que j'étais encore à l'hôpital, en décembre 1952.

D.S. Thério, c'est un nom d'origine acadienne? 
A.T. Oui, ma famille est d'origine acadienne. L'ancêtre est arrivé à Grand-Pré, Nouvelle-Écosse, en 1637. Mais ce n'est peut-être pas vrai pour la famille de Yves Thériault car il y a aussi une famille canadienne de Thériault.

D.S. Mais vous venez de dire que vous êtes parent avec Yves Thériault !

A.T. C'est vrai, mais pas nécessairement de la même famille de Thériault. Son grand-père Thériault était marié à une Thériault qui était la sœur de mon grand-père Thériault. C'est donc par sa grand-mère que nous sommes parents. Son père et le mien sont cousins germains.

D.S. Vous avez été directeur du Département de français au Collège militaire de Kingston. J'ai toujours admiré le fait que vous ayez pu attirer un nombre impressionnant de spécialistes québécois à Kingston. Léandre Bergeron, Normand Leroux, Arsène Lauzière, François Gallays, Guy Plastre, Gérard Bessette et bien d'autres Québécois. Comment avez-vous pu accomplir cet exploit, car dans la plupart des universités ontariennes, il y a très peu de Québécois?

A.T. Je n'ai aucun mérite, dans le fond, puisque je n'ai fait que continuer la politique de mon prédécesseur, Léopold Lamontagne. II cherchait des Québécois et il en trouvait. J'en ai cherché moi aussi et j'en ai trouvé. Nous étions un petit groupe de douze dans ce département au moment où je suis parti pour I'Université d'Ottawa.

D.S. Vous avez donné des cours de création littéraire à l'Université du Québec, à Trois-Rivières et à I'Université d'Ottawa. Parlez-nous un peu de cette expérience.

A.T. On n'enseigne pas l'art d'écrire. On incite des étudiants, qui ont envie de devenir écrivains, à écrire. Evidemment on doit parler théorie dans ces cours mais la partie théorique doit normalement être un échange avec les étudiants. C'est un cours où on force les étudiants à découvrir leurs possibilités. Il faut leur dire: allez-y, n'ayez pas peur. Ce n'est pas toujours facile. Le cours que j'ai donné à Trois-Rivières a été l'occasion d'échanges stimulants avec les étudiants pendant la partie théorique. J'ai eu d'autres cours où les étudiants ne parlaient presque pas. Dans ces cas-là, ca devient difficile.

D.S. Vous avez écrit trois livres sur Jules Fournier, journaliste et pamphlétaire. II y a eu d'abord votre thèse de doctorat, Jules Fournier, journaliste de combat (1955), puis Jules Fournier, publié chez Fides en 1957, et enfin, en 1964. Mon encrier de Jules Fournier, également chez Fides. Qu'est-ce qui vous a tellement fasciné chez Fournier? Son nationalisme fondé sur des idées économiques qui ne sont pas sans liens avec la politique 
sociale-démocrate du P.Q., son désir de justice sociale, son encouragement donné aux auteurs d'ici, ou peut-être son style ironique, clair, toujours précis?

A.T. Je ne saurais exactement vous répondre. C'est Luc Lacourcière qui m'a fait découvrir Fournier et qui m'a proposé d'en faire le sujet de ma thèse. Mais ce que je puis dire, c'est que je suis entré dans l'œuvre de Fournier comme on entre chez quelqu'un avec qui, au premier contact, on s'entend merveilleusement. Sa façon directe de dire les choses, son désir de justice ou ses dénonciations d'injustices, son humour, son ironie, sa façon de se mettre au blanc, de risquer l'opprobre général pour mettre un peu de sens dans la tête de certaines gens que le pouvoir rendait loufoques, c'est un peu tout cela qui m'a plu chez lui. J'ai fait cette thèse sur Fournier à vingt-cinq ou vingt-six ans. J'avais peu d'expérience, à ce moment-là. Il me semble que j’aurais pu faire un meilleur livre, avec quelques années de plus de fréquentation de la littérature. Mais il fallait que je gagne ma vie. Et je suis parti la gagner, après mes études à Harvard, au Collège Bellarmin de Louisville, Kentucky, parce qu'il n'y avait à peu près pas de débouchés dans les collèges classiques, à ce moment-là. J'y enseignais le français. Deux ans après, en 1956, je passais à l'Université Notre-Dame, en Indiana, où je donnais des cours de français et de littérature française. J'ai été à Notre-Dame trois ans, à Tóronto un an avant d'arriver à Kingston, au Collège militaire, où je suis resté neuf ans. J'ai été directeur de leur Département de français pendant sept ans. C'est en arrivant à Kingston que j'ai eu l'idée de fonder Livres et Auteurs, une idée qui devait me trotter dans la tête depuis quelques années.

D.S. Votre expérience comme directeur de Livres et Auteurs vous a sûrement apporté beaucoup de satisfactions, mais aussi des déceptions.

A.T. Des satisfactions oui. L'idée principale, c'était de faire connaître les écrivains d'ici. J'ai toujours eu l'impression, en lisant journaux et revues, et je l'ai encore, que les laissés pour compte ont toujours été les écrivains. Si des écrivains écrivent, il me semble normal qu'on parle d'eux quelque part.

Je ne sais pas si je peux parler de déceptions. J'ai eu beaucoup de difficultés à maintenir Livres et Auteurs en vie. J'ai investi pas mal d'argent dans cette entreprise. Les seuls numéros avec lesquels je n'ai pas perdu d'argent, ce sont les numéros 1970, 1971 et 1972. II $\mathrm{m}$ 'a donc fallu attendre neuf ans avant qu'on me donne les subventions nécessaires pour faire vivre l'entreprise.

Je l'ai vendue à l'Université Laval au moment où je me suis rendu compte, avec le nombre croissant de livres publiés chaque année, que je ne pouvais continuer à faire toute la besogne que je devais faire comme directeur général. II fallait une équipe et un secrétariat. Qui m'aurait fourni tout cela? Les gens de Laval étaient prêts à prendre la relève. 
C'est arrivé au bon moment puisque, en 1973, je me sentais très malade sans trop savoir ce que j'avais. Je suis entré à l'hôpital quelques mois plus tard pour une hernie hiatale. J'ai tout de suite été opéré. On ne l'aurait probablement pas fait si on avait su que la hernie n'était pas mon seul mal. J'ai dû passer presque deux mois à l'hôpital et j'ai mis deux ans à me remettre complètement.

D.S. Et puis après Livres et Auteurs, c'est une revue de l'actualité littéraire que vous avez fondée: Lettres québécoises. Vous n'avez pas eu la vie facile en tant que directeur.

A.T. Non, la vie n'a pas été facile à Lettres québécoises depuis les débuts et elle ne l'est pas encore. J'espérais en quelques années faire comprendre aux pouvoirs publics que cette revue nous était nécessaire. Je n'ai pas encore réussi. Je ne suis donc pas certain de l'avenir de cette revue. II me semble indécent qu'on m'oblige encore, après ces cinq années d'existence, à renflouer la barque à coups de milliers de dollars. Je commence à $m$ 'habituer un peu à l'incompréhension des gens en place. Je dis cela et, malgré tout, je ne m'y habitue jamais.

D.S. Vous croyez passionnément à la civilisation québécoise. Tout comme le narrateur de votre Soliloque en hommage à une femme, vous voulez la faire connaître au public. Depuis déjà trente ans, vous avez publié des critiques et des anthologies sur des écrivains, des journalistes, même sur des hommes d'État et d'Église. Vous avez contribué énormément au rayonnement de notre civilisation.

A.T. Chaque peuple a une civilisation à lui. Et je fais ici une différence entre civilisation et culture. La civilisation c'est ce qui nous vient des parents, de l'entourage, du milieu, des façons de vivre, de se comporter, etc. La nôtre, en Amérique du Nord, est très spéciale et je dirais que, pour la comprendre, il faut absolument retourner au dix-neuvième siècle surtout, même au dix-huitième et plus loin. Nous sommes les héritiers d'une façon de penser qui nous vient directement du dix-neuvième siècle. Nous aurions pu, comme peuple, être très différents de ce que nous étions en 1960, si le libéralisme du dix-neuvième siècle avait gagné la partie. Ce n'est pas le cas et c'est dommage. Cela ne veut pas dire, même si nos origines sont modestes, même si nos ancêtres ont été brimés intellectuellement par les autorités en place, que nous devons fermer la porte à ce passé. Au contraire. Il faut nous accepter comme nous sommes et tâcher de devenir autres, de nous transformer.

D.S. Pourquoi avoir publié des anthologies de conteurs et d'humoristes?

A.T. Tout simplement parce que ça n'existait pas. J'ai fait les conteurs québécois de 1940 à nos jours parce que c'était une période que je connaissais assez bien. L'anthologie sur l'humour m'a donné plus de mal. J'ai dû fouiller les archives, mais je suis sûr que j'ai oublié des choses importantes que d'autres découvriront. J'ai fait plusieurs trouvailles aussi. 
D.S. En 1975, vous avez publié, avec deux autres critiques, Ignace Bourget, écrivain. L'ancien évêque de Montréal est une figure centrale dans l'organisation de la société au dix-neuvième siècle. Vous avez prouvé qu'il était aussi un écrivain efficace. Un peu comme dans le cas de Jules Fournier, vous êtes allé déterrer l'œuvre d'un homme d'action dont vous appréciiez les talents d'écrivain. Vous avez une conception très large de la littérature.

A.T. Evidemment, quand on pense à la littérature, on pense d'abord à la création. On pense au roman, à la poésie, au théâtre. Mais l'éloquence est aussi une forme de création. Et l'éloquence politique et nationaliste a fleuri au XIXe siècle. Bourget a certainement été efficace dans ses écrits parce qu'il disait clairement ce qu'il a vait à dire et il avait, en plus, les moyens d'atteindre ceux à qui il destinait sa prose. Cela ne fait pas nécessairement de lui un grand écrivain. II reste cependant que certains de ses mandements, à cause de l'élégance du discours et la charge d'émotion qu'ils contiennent, méritent de faire partie des meilleurs textes d'éloquence du dix-neuvième siècle.

D.S. Vous vous êtes intéressé à des polémistes comme Fournier et Asselin. Et je sais qu'Adrien Thério, professeur, aime parler de l'esprit critique d'Arthur Buies. II est donc tout à fait normal que vous soyez vous-même polémiste. Dans vos éditoriaux, vous attaquez avec à propos ceux qui nuisent à l'épanouissement de la culture québécoise: des éditeurs sans conscience nationale; un Conseil des arts insensible à la question de la culture nationale; la Société Radio-Canada qui néglige les auteurs d'ici...

A.T. Je crois que j'attaque surtout ceux qui prétendent travailler à l'épanouissement de la littérature et de la culture pour bien se faire voir du public, et aussi ceux qui pourraient faire quelque chose pour les arts en général et qui ne font rien. Quand au Conseil des arts du Canada, toute sa politique est à revoir. Pour éviter la sclérose, il faudrait que les personnes qui détiennent les postes importants du Conseil aient un mandat bien défini de trois ou quatre ans.

D.S. En 1975, vous avez publié Des choses à dire, journal où vous attaquez certains intouchables du monde de l'édition, et où vous vous laissez aller à des commentaires fantaisistes sur des auteurs comme Bessette et Ferron. Et puis, quelques mois après la parution du livre, vous l'avez retiré du marché. Pourquoi?

A.T. Parce que les trois premiers lecteurs qui m'en ont parlé m'ont dit que j'avais été très dur pour Bessett dans l'entrevue imaginaire que je faisais avec lui. Je croyais que c'étaiı seulement ironique et drôlatique. Les lecteurs dont je parle ne l'ont pas vu du même œil. Je me suis dit que je n'avais aucune raison d'attaquer Bessette, qui est un de mes amis, et j'ai donc retiré le livre du marché.

Je sais que des gens ont raconté que j'avais peur de me retrouver en 
justice pour avoir inclus dans le livre une lettre de Pierre Tisseyre, sans sa permission. La permission, je l'avais bel et bien obtenue de $M$. Tisseyre. II s'agissait donc de racontars.

D.S. Vous avez fondé votre propre maison d'édition. Ainsi depuis 1973, vous publiez tous vos romans chez Jumonville.

A.T. J'ai fondé ces éditions vers 1966 . Je voulais que Livres et Auteurs révèle d'une maison d'édition. Par la suite, j’ai décidé d'y publier mes romans. Ce n'était peut-être pas une bonne idée parce que les chroniqueurs littéraires, en général, portent bien peu d'attention à tout ce qui vient des maisons d'édition de ce genre. La preuve, mon dernier roman: C'est ici que le monde a commencé, n'a eu que deux comptes rendus. Je suis toujours persuadé qu'il méritait un bien meilleur traitement.

D.S. Est-ce que vous gardez certaines amertumes, déceptions ou même colères suite aux luttes que vous avez menées pour promouvoir la littérature québécoise?

A.T. Que voulez-vous que je vous dise? Vous me posez cette question au moment où les autorités du Conseil des arts s'entêtent à ne pas nous subventionner sur le même pied que d'autres. Je suis convaincu qu'on a tout fait, indirectement, pour faire mourir cette revue. II y a des gens qui croient que j'invente des drames. II faudrait une enquête sur toute cette affaire. On finirait peut-être par mieux comprendre.

D.S. On vous a parfois accusé de voir des complots partout, d'exagérer, d'attaquer trop souvent des individus au lieu de vous en prendre aux institutions. Qu'en dites-vous?

A.T. Je n'ai jamais parlé de complots moi-même. Je n'en ai jamais été témoin. Mais j'ai vu des injustices. Par exemple, entre 1962 et 1981, un seul de mes récits ou romans a fait l'objet d'une critique dans le Devoir. C'est André Major qui a parlé du Soliloque. La raison de tout cela? J'ai publié un article dans /e Devoir en 1961 où je m'en prenais au jugement porté par Jean-Éthier Blais sur quatre ou cinq livres dont il venait de parler. On a donc décidé de faire - cela m'a été rapporté - comme si je n'existais pas. Les deux autres chroniqueurs littéraires du temps, Jean Basile et Jean Hamelin, ont dû être d'accord puisqu'ils n'ont jamais fait de critiques de mes récits. II est bien possible que Jean Basile n'ait jamais été intéressé par mes romans et c'est son droit. Mais Jean Hamelin que j'ai rencontré par hasard et à qui j'ai demandé, quelques mois après la sortie de Mes beaux meurtres, s'il allait en parler, m'a répondu qu'il était trop tard et que par ailleurs, comme j'avais attaqué un de ses confrères... Ces chroniqueurs auraient pu refuser de parler de mes livres mais pourquoi n'ont-ils pas demandé à quelqu'un de l'extérieur de le faire?

C'est ce que j'ai fait à Livres et Auteurs. J'ai fait des efforts pour trouver de bons critiques pour parler des livres de ces trois écrivains. J'ai moi- 
même fait des articles très favorables sur deux livres de Jean Hamelin et je l'ai inclus dans mon anthologie Conteurs canadiens-français. Pour moi, l'individu et l'écrivain, c'est deux choses différentes.

La politique du Devoir ne doit pas avoir tellement changé puisqu'on n'a pas jugé bon, depuis 1974, de parler de La Colère du père, de La Tête en fête ni de C'est ici que le monde a commencé.

Je ne suis tout de même pas pour vous dire que ça me plaît d'avoir été mis de côté, systématiquement, comme ça. J'en retiens ceci, c'est que, quand on a un petit peu de pouvoir, dans le monde littéraire d'ici, on le défend si jalousement que les échanges deviennent impossibles.

Avant de passer à la dernière partie de cette entrevue, quelques commentaires sur l'œuvre de l'écrivain s'imposent. Adrien Thério a déjà publié quinze cuvres de fiction, dont des contes et nouvelles, des récits, des pièces de théâtre et des romans. Les sujets préférés de l'auteur peuvent se diviser en deux catégories principales : récits, romans et journaux intimes ressuscitant soit le Québec des rangs et des concessions pendant les années trente (six œuvres en tout), soit la vie des cloîtrés (deux œuvres) ou des professeurs (cinq); une série de contes fantastiques, macabres, cauchemardesques. souvent d'une violence déchaînée (deux recueils). II y a donc chez Thério deux tendances fondamentales, deux courants ou manières: le raconteur d'histoires qui apparaissent surtout sous forme de journal ou de récit; et l'interprète du subconscient, des pulsions intérieures. Ces tendances s'entremêlent, le raconteur se laissant aller inévitablement à des rêveries, à des visions intenses, saisissantes (appelées «fantasmes", "cinéma intérieur", ou tout simplement «rêves») où sont mises en scène les obsessions privilégiées de l'auteur telles que l'érotisme, la colère sous toutes ses formes, l'amour de la nature.

Deux paysages ou décors dominent dans l'œuvre de Thério: paysage onirique d'hallucinations, de «spectacles étranges de certains de nos rêves" (Mes beaux meurtres, p. 148); paysages descriptifs, foncièrement réalistes, du Chemin Taché et de ses environs. Voici le Chemin Taché:

Le Chemin Taché m'entourait avec ses grands champs de neige où des souches apparaissaient ici et là. la côte du nord dont le haut était recouvert par la forêt et toutes ces maisons en grosses pièces de bois équarries posées les unes par dessus les autres, la plupart flanquées $d^{\prime} u n$ bout de grange qui attendait le rajout qu'on se promettait de faire d'été en été. A intervalles réguliers, des clôtures de pieux qui se perdaient au loin dans un commencement de forêt. Tout mon horizon, en fait, au sud, au nord, à l'ouest était bouché par la forêt quireculait de quelques arpents chaque année. Ici et là une sorte d'éclaircie qui permettait de 
voir plus loin. Mais la route était bordée d'habitations des deux côtés et donnait l'impression de vouloir devenir un village. Un magasin général, une école. un forgeron, une boutique de menuiserie, un bureau de poste, une scierie du côté de Saint-Hubert, nous manquait-il quelque chose? Je savais que chaque maison était vivante, qu'un poêle à deux corps mettait de la chaleur partout. Je regardais toutes ces choses avec des yeux neuts, je touchais à tout cela avec mon regard, et, heureux probablement de me sentir vivre avec tout ce qui vivait autour de moi, je me mis à imaginer que le Chemin Taché n'était qu'un petit enclos que je soulevais d'une main et que je faisais tourner dans lair sec comme s'il se fut agi d'un objet quelconque avec lequel je me serais amusé. (La Colère du père, p. 69-70)

II est évident que le Thério qui parle ici aime les choses et les gens de la campagne. Toutefois, il ne prêche jamais le retour à la terre. Il nous dit au contraire qu'il faut quitter son petit patelin pour vivre dans un monde intellectuellement stimulant, mais, paradoxablement, on doit toujours «retourner au clan", à la famille et aux paysages de l'enfance. Chez Thério, on ne renie jamais ses origines «petites-villageoises». On reste déchiré entre la ville et la campagne.

Adrien Thério excelle à camper trois types de personnages. D'abord, l'adolescent, individu presque toujours malheureux, incompris de ses parents et de ses amis, désireux d'apprendre tout sur la vie, découvrant la beauté de la nature ainsi que les joies et les déceptions de l'amour. Ensuite, le personnage du père, être insensible et autoritaire. Enfin, les différents professeurs, celui qui se sent mal à l'aise dans le monde prétentieux des intellectuels, ou celui qui manque d'imagination, qui ne remet rien en question, ou celui qui, tout au contraire, aspire à se dégager du lieu commun:

De la couleur, de la couleur avant toute chose. Tout est si terne. Tout est si ennuyeux. Les étudiants vont en classe pour avoir un papier qui leur permettra de gagner de l'argent. Les professeurs vont en classe pour avoir le droit de dépenser le chèque qui arrive tous les mois. Des chercheurs font une thèse de doctorat pour obtenir plus d'argent et se taisent ensuite. Personne ne pense à mettre de la couleur dans la vie. Un païen chez les pingouins, $p$. 123)

Les narrateurs de l'œuvre de Thério ont plusieurs points en commun. Ils s'opposent aux valeurs bourgeoises, au fanatisme religieux, ou capitaliste, a l'apathie. Ils réclament, tant pour l'homme que pour la femme, l'égalité dans la famille et dans le travail. Le monde selon Adrien Thério est dépourvu de valeurs absolues. L'égoïsme s'avère l'ennemi constant, peut-être inévitable, de l'homme. Mais tout n'est pas noir. Loin de là! ll y a ces moments privilégiés, éphémères il est vrai, où règnent l'amour, l'amitié, et l'attachement sensuel au pays de l'enfance. L'amour de la nature, le merveilleux mystère des bois, l'appel des montagnes et des rivières, viennent donner, sinon un sens à la vie, du moins une raison de continuer. C'est que Thério est poète. L'arbre devient enracinement, jouissance. Les personnages «sentent le besoin de se coller à la terre" (Les Fous d'amour, p. 46), de se laisser bercer par les pouvoirs magi- 
ques de la neige.

Adrien Thério sait raconter une histoire, sait décrire d'une façon réaliste des lieux et des personnages. Heureusement, il sait également délirer, traduire d'une façon originale et personnelle l'attachement sensuel au pays, sans oublier nos fantasmes, érotiques ou traumatisants, tendres ou cruels. Allons maintenant le questionner sur cette œuvre qui réservera d'agréables surprises à ceux ou à celles qui ne la connaissent pas encore.

D.S. Dans Un paien chez les pingouins, vous écrivez: «La littérature a-t-elle une utilité quelconque? Je pourrais répondre non et n'aurais sans doute pas tort. Je peux dire aussi que, sans elle, la vie manquerait de couleurs. La couleur, c'est le pouvoir de transformation, le pouvoir de rendre plus vivant» (p. 115). Voulez-vous expliquer davantage votre conception de la littérature?

A.T. Mon Dieu! la littérature, c'est la passion sous toutes ses formes. C'est l'être humain tout entier qui lutte contre les forces du mal et les forces de la nature. C'est cela qui doit transparaître à travers l'écriture. C'est la vie elle-même qu'on ressuscite. La littérature ne rend personne meilleur ni plus moral. Elle permet cependant de mieux vivre sa vie, de nous inventer un monde à notre mesure pour échapper à celui qui ne nous satisfait pas. Et ceci est aussi vrai de celui qui écrit que de celui qui lit. L'amateur de romans policiers ne fait pas autre chose que chercher, de livre en livre, le genre de vie dont il rêve et que le monde dans lequel il vit ne peut lui donner.

D.S. Pourquoi avez-vous écrit, au tout début de votre carrière, des contes pour adolescents?

A.T. Je n'ai vraiment pas pensé à écrire des contes pour adolescents. J'ai publié des contes dans des revues et journaux pour me faire de l'argent de poche quand j'étais étudiant. Par la suite, j'ai cru qu'un certain nombre d'entre eux conviendraient à un public jeune. Je les ai réunis dans Contes des belles saisons.

D.S. Lorsque vous évoquez, dans Contes des belles saisons (Beauchemin, 1958), I'histoire du jeune garçon solitaire dupé par ses camarades de classe, ou du jeune Hermann au père insensible, est-ce un peu de vousmême dont vous parlez?

A.T. L'histoire du garçon solitaire dupé par ses camarades, je ne m'en souviens pas du tout. Quant à l'histoire d'Hermann, elle $m$ 'a été inspirée par un jeune étudiant à qui j’ai enseigné pendant ma première année, en Éléments latins, à Québec, qui s'appelait justement Hermann et qui, 
semble-t-il, se faisait solidement rosser par son père. II ne doit pas me ressembler parce que mon père battait rarement ses enfants. Ma mère l'en empêchait.

D.S. Flamberge au vent (Beauchemin, 1962), roman pour adolescents, semble être pour vous l'occasion de jouir d'un de vos passe-temps favoris: être près de la nature et des animaux.

A.T. Cette histoire m'a été inspirée par un de mes amis qu'on avait expédié à la campagne, chez une tante, avec son frère et sa sœur, pour ne pas les avoir sur les bras, au magasin général. II est bien possible que ces personnages soient près de la nature. C'est tout à fait inconscient.

D.S. Vous êtes, avec Victor-Lévy Beaulieu, qui vient de Saint-Jean-de-Dieu, et Roger Fournier, qui est né à Saint-Anaclet, un des porte-parole littéraires les plus importants du Bas du fleuve. Parlez-nous un peu de quelques-uns des personnages qui vous ont servi de modèles pour votre Chemin Taché.

A.T. Je n'ai jamais pensé à aller chercher des modèles chez les gens que je connaissais. Je m'inventais des histoires et après, je suppose qu'instinctivement je choisissais des personnes de mon entourage pour créer mes personnages. Je me rends compte que, en agissant ainsi, j'ai utilisé un grand nombre de personnes du Chemin Taché et des alentours. Par exemple, le clan des trois frères qui dirige plus ou moins le Chemin Taché dans La Colère du père et dont la tête dirigeante est le père du narrateur qui a douze ans. Eh! bien, j'ai inventé mon histoire et j'avais besoin de ce clan, de ces trois frères pour bien mener l'affaire. Pour mieux les faire vivre, je leur ai donné l'apparence d'un de mes oncles Lebel et de ses deux frères, nom que j'ai changé en Martel. J'ai gardé leurs prénoms tels quels. Les trois frères de $\mathrm{La}$ Colère du père finalement $\mathrm{n}^{\circ}$ ont rien à voir avec mon oncle et ses frères. Ils leur ressemblent physiquement et moralement, c'est tout. Je mets donc quelqu'un que je connais dans la peau d'un personnage que j'invente pour me faciliter la tâche.

Prenons un autre exemple: il n'y a pas un personnage dans Ceux du Chemin Taché qui ait vraiment existé. Seulement, je crois que ces gens sont tous du Chemin Taché, par leur façon de se conduire, de vivre.

Quelques-uns de mes personnages ressemblent un peu à mon père mais finalement ce n'est jamais lui.

D.S. Les Brèves Années, publié chez Fides, est un roman dont les personnages clefs sont des adolescents, mais ce n'est pas un roman réservé aux adolescents?

A.T. Je ne crois pas, non. C'est un récit où la réalité et la fiction sont si bien entremêlées que je ne saurais moi-même dire ce qui fait partie de mon histoire et ce qui est fiction. Ce livre évidemment rappelle Le Grand Meaulnes que j'avais lu à dix-sept ou dix-huit ans. J'en avais vingt-cinq 
quand j'ai écrit Les Brèves Années. Dans les deux romans, les personnages sont des adolescents. Il est donc facile de dire que j'ai été influencé par le livre d'Alain-Fournier. II est possible aussi que, sans m'en rendre compte, j'aie puisé chez lui des idées, des recettes. C'est la deuxième partie de mon récit qui fait penser au Grand Meaulnes à cause du Grand Bois, de la Route inconnue, du pays sauvage. Mais je n'ai pas eu besoin d'aller chez Alain-Fournier pour trouver ces lieux. Ils existent bel et bien au quatrième rang de Saint-Hubert où nous avions notre terre à bois. J'y suis retourné il y a deux ans. J'en ai rapporté de belles photos. Le pays est encore plus sauvage qu'il l'était quand j'étais jeune.

D.S. Les Brèves Années est, du moins en partie, un roman de mceurs. Vous y évoquez des jeux d'enfants inconnus des gens de la ville. Vous décrivez l'organisation sociale fondée sur les paroisses et sur les rangs. Vous ressuscitez le milieu des collèges classiques (transformant deux collèges que vous avez fréquentés, le Séminaire de Rimouski et le Collège SaintAlexandre, à Limbourg). On pourrait donc qualifier une partie importante de votre cuvre de roman social.

A.T. C'est bien possible, mais je n'ai jamais pensé à cela.

D.S. Dans Les Brèves Années, Clair Martin s'inquiète du sort réservé au patrimoine. Menaud, maître-draveur est son livre préféré. Vous avez eu Félix-Antoine Savard comme professeur. L'auteur de Menaud a-t-il eu une influence importante sur vous?

A.T. J'ai relu deux chapitres des Brèves Années il y a quelques mois et j'ai eu la surprise de ma vie d'entendre Clair Martin parler de Menaud. J'avais complètement oublié cela. C'est un fait que Menaud, au début, m'a beaucoup remué. Je vous ai dit tout à l'heure que j'avais fait un récit dans le même genre, récit que j'ai détruit, heureusement.

D.S. Qui sont les autres auteurs, québécois, français ou américains, qui vous ont influencé?

A.T. Je ne saurais dire s'il y a des auteurs qui m'ont vraiment influencé. II y a un bon nombre d'écrivains d'ici et d'ailleurs que j'aime beaucoup, par exemple Gabrielle Roy, Yves Thériault, Marie-Claire Blais; chez les étrangers, je nommerais Marcel Proust, Albert Camus, Julien Green, Dostoïevski, Faulkner, Steinbeck, D. Thoreau, Herman Hesse. Vous voyez qu'il y a un monde de différence entre les uns et les autres et il n'y a rien chez moi qui leur ressemble. Tirez vos conclusions.

D.S. Les Brèves Années n'est que la première tranche de I'histoire des habitants du Chemin Taché, les autres étant «Le Chat sauvage" (dans Mes beaux meurtres), Le Printemps qui pleure, Ceux du Chemin Taché, La Colère du père et C'est ici que le monde a commencé. Toutes ces œuvres, à l'exception du Printemps qui pleut (qui me semble artificiel et répétitif), renferment des dialogues naturels, des descriptions vivantes et justes, des rêveries et des hallucinations fascinantes. Prenons par exemple les 
contes de Ceux du Chemin Taché, histoires étranges d'un violoneux enchanteur, de fous, de revenants et de tireuses au thé. Est-ce que vos contes sont, au point de départ, inspirés de la tradition orale, de contes que vous avez entendus pendant votre jeunesse, ou est-ce que vous avez surtout inventé les anecdotes de Ceux du Chemin Taché?

A.T. Je n'ai jamais entendu de contes pendant ma jeunesse. Je me souviens qu'à l'âge de douze ou treize ans, je me délectais pendant les soirées d'hiver à lire des histoires de pirates que j'avais découvertes dans des grandes revues trouvées je ne sais plus où. J'ai fouillé dans ma mémoire pour savoir d'où ces revues, bien illustrées, en brun je crois, provenaient. Je n'ai rien découvert.

En fait, j'ai eu envie de raconter des histoires moi-même tout de suite après avoir lu mon premier récit, à dix ou onze ans, livre que j'avais reçu en prix à la fin d'une année scolaire et qui s'appelait Le Petit Violon de la grande demoisel/e. J'ai donc inventé des histoires en me basant sur ce que je voyais autour de moi. L'épisode du violoneux qui fait danser tout le monde dans $L a$ Colère du père, et avec lequel je fais une promenade dans les airs, me vient directement de notre voisin Alphonse Ouellet qui était un excellent violoniste (dans ma tête de dix ans) et qui, en plus de jouer du violon sur sa galerie le soir, venait souvent chez nous avec son instrument. J'avoue que cela m'enchantait. Je ne comprenais pas qu'on puisse tirer des sons pareils de cet instrument. J'ai eu besoin de quelqu'un pour faire danser les gens dans $L a$ Colère du père puisque les gens du Chemin Taché, devenus protestants, pouvaient maintenant danser et, le connaissant bien, je l'ai invité à jouer. Et je lui ai fait faire une belle promenade au-dessus du Chemin Taché.

D.S. Dans La Colère du père (Jumonville, 1974), un drame éclate au Chemin Taché: privés d'église par un évêque entêté et cérémonieux, les habitants du rang se font protestants. Ce roman m'a beaucoup plu. Vous avez le sens de l'anecdote bien menée, du suspense, de l'humour simple et vrai des petites gens. Y a-t-il du vrai dans l'histoire de la conversion des gens du rang à la religion protestante?

A.T. Quand je suis arrivé au Chemin Taché, à l'âge de cinq ans, il n'y avait plus de protestants. Mais une vingtaine d'années plus tôt ou un peu plus, ils étaient tous devenus protestants pour la raison que j'ai donnée dans le récit. II restait encore, à mon époque, des vestiges de ce schisme. L'emplacement du cimetière protestant, la maison qui avait servi de mitaine. Et les gens parlaient encore de ce dérangement. Finalement, l'envie m'est venue de raconter une histoire semblable. Je me disais: comment peut-on devenir subitement protestant quand on a été catholique depuis toujours? Cela a dû perturber les consciences. C'est ca que j'ai voulu montrer en recréant les principaux personnages du drame et en leur laissant toute liberté d'action. J'ai moi-même été surpris de leurs actions et de leurs paroles, à certains moments. 
D.S. Dans plusieurs de vos œuvres, le père est un «chef de clan», un homme orgueilleux et sec qui ne veut pas que ses enfants aillent au collège. Pour lui, c'est la terre et le travail manuel qui comptent. Y a-t-il quelque chose d'autobiographique dans toutes ces «colères du père»?

A.T. Il y a des traits de mon père dans quelques-uns de mes personnages. Le père sec et autoritaire que vous venez de décrire existe dans certains de mes livres mais je vous dirai par ailleurs que mon père n'a jamais voulu m'empêcher d'aller à l'école. Et il n'a pas mis d'obstacle à ce que j'aille au collège. Je me souviens même qu'un jour il est venu prendre ma place, alors que je travaillais à retourner du grain, dans les champs, parce qu'il venait d'apprendre par ma mère que je ne voulais pas manquer l'école. II m'a pris la fourche des mains et $\mathrm{m}^{\prime}$ a dit: "Va-t'en, à ton école!"

D.S. Vous avez une prédilection pour les récits. Tous vos romans, ou presque, sont écrits à la première personne, et La Colère du père ne fait pas exception.

A.T. Je me sens plus à l'aise quand je raconte les choses au «je». Je crois que mon premier roman, La Soif et le Mirage, est écrit à la troisième personne. Ce n'est pas un très bon roman. L'important avant da me mettre au travail, c'est que je réussisse à devenir, psychologiquement parlant, le personnage qui deviendra le narrateur. Je me suis fait moine cloîtré dans Les fous d'amour. Certaines personnes ont pensé que j'avais fait un séjour chez les moines. Je ne suis jamais entré en communauté. J'y ai songé à l'âge de quinze ans. Mais je n'y songeais plus à vingt ans. Psychologiquement parlant, je suis entré chez les moines, un an avant de commencer à écrire Les Fous d'amour, pour permettreà mon narrateur de bien jouer son rôle.

D.S. Le volet le plus récent des gens du Chemin Taché s'intitule C'est ici que le monde a commencé (Jumonville, 1978). Le titre indique bien l'importance de ce petit rang du Bas du fleuve dans votre cuvre.

A.T. Le monde commence toujours là où on a ses racines. Et mes racines, elles sont là dans ce pays du Chemin Taché que j'ai agrandi un peu pour $y$ inclure toute la paroisse de Saint-Cyprien que je préfère appeler SaintAmable, le Grand Bois qui se trouve situé dans Saint-Hubert, et SaintÉpiphane et Saint-Modeste, les paroisses tout près de Rivière-du-Loup qui ont vu naître mon père et ma mère.

C'était alors un pays pauvre, comme beaucoup d'autres pays ruraux, et c'est, je crois, cette pauvreté qui a été la cause de bien des drames que j'ai vus et que je n'ai pas fini de raconter. J'ai ressenti très jeune les effets de cette pauvreté. Je crois que j'en ai été humilié, chaque fois que mon père l'a été, pour toutes sortes de raisons.

D.S. C'est ici que le monde a commencé est selon moi le meilleur livre du cycle des gens du Chemin Taché. Vous êtes tellement à I'aise lorsque 
vous évoquez, tout en le transformant, bien sûr, l'univers de votre enfance. La sexualité joue un rôle prépondérant dans C'est ici que le monde a commencé. Le narrateur a la sexualité en éveil. C'est un hédoniste avoué (génital, oral, anal), et un bissexuel en puissance. Il y a énormément d'exemples d'ambivalence sexuelle dans votre œuvre. Lorsque Clair s'éprend de Solage (Les Brèves Années). Jacques devient jaloux et se bat avec son ami. Le jeune professeur de La Soif et le mirage aime autant Bill que Mary Lane. La belle-mère dans \& Le Chat sauvage» accuse son petit-fils de ne pas être attiré par les filles. Et nous devinons facilement que le narrateur de Soliloque en hommage à une femme est paradoxalement bissexuel. Dans Un paien chez les pingouins, un étudiant épouse une fille, par devoir familial, mais aime secrètement un homme. Et puis il y a tous ces symboles masculins dans votre cuvre: le soleil, l'arbre, les clochers.

A.T. Je ne me souviens pas de la bataille de Clair avec Jacques. Plusieurs autres faits que vous rapportez me sont complètement sortis de la mémoire. Dans l'ensemble, cependant, je crois que vous avez vu juste.

Vous savez, toutes les religions ont perverti l'amour. Elles ont édicté toutes sortes de règlements et nous ont affirmé qu'en dehors de leurs noms, tout le reste est péché. Que la majorité de la population ait accepté pendant des milliers d'années ces normes stupides me renverse. Tout amour est beau et personne n'a le droit de le détruire ou de le défendre. Croire qu'il y a un amour normal et un autre anormal est une aberration de l'esprit. Et ce que j'essaie de dire, à ce sujet, dans mes livres, c'est qu'il faut sortir de ce carcan imposé au cours des siècles par les plus grands frustrés de la Terre. C'est l'amour, c'est le désir sexuel qui transforme toute la vie. Brimer ses élans, c'est brimer ce qu'il y a de plus beau en soi.

Les symboles du soleil, de l'arbre et des clochers, je ne m'y suis jamais arrêté. II doit y en avoir d'autres aussi.

D.S. II y a une scène qui revient continuellement dans vos romans et qui n'est pas sans connotation sexuelle: des jeunes, fascinés par une rivière, se baignent tout nus et jouissent mystérieusement des effets bénéfiques de l'eau.

A.T. Je ne sais pas d'où cela vient. Je me souviens que jeune, je me suis baigné nu, même si je ne savais pas nager, dans la rivière que j'appelle la Blonde et qui porte un autre nom moins poétique, au Grand Bois, et dans cette même rivière tout près du village de Saint-Amable, et que j'en retirais un grand plaisir.

D.S. Vos adolescents sont à la fois naïfs et grivois. Ils s'émerveillent comme des enfants face à la nature, aux chevaux, à la pêche. Puis ils font l'amour comme des déchaînés. 
A.T. A quel endroit font-ils l'amour comme des déchaînés? Je ne m'en souviens pas. Mais dans le fond, c'est tout a fait normal. C'est quand on s'ouvre aux mystères de l'amour qu'on a le plus besoin de faire l'amour.

D.S. Passons maintenant à vos œuvres où le narrateur est un professeur. En 1960, c'était $L a$ soif et le mirage, roman humoristique constitué d'observations sur la civilisation américaine des années cinquante. En 1965 , c'est un professeur de collège classique à l'humour de pincesans-rire qui nous livre, dans Le Mors aux flancs, ses observations sur la société canadienne-française. Et enfin, en 1970, un professeur, «païen chez les pingouins", nous décrit cette «bande de moutons noirs à fourrure blanche" que sont les professeurs d'université. Tous ces narrateurs ont le sens de l'humour. Le Québec n'a pas beaucoup d'humoristes, et vous affirmez dans la préface du Mors aux flancs que plusieurs éditeurs ont refusé de publier ce roman parce que «le public canadien-français n'est pas habitué à rire».

A.T. Dans le fond, Le Mors aux flancs n'est pas un très bon roman. Mais il y a certains chapitres qui sont vraiment drôles, je crois. C'est là son principal intérêt. Je n'ai pas envie de relire La Soif et le Mirage parce que c'est mon plus mauvais livre. Y a-t-il de l'humour là-dedans, je ne sais. Dans Un paien chez les pingouins, il y a peut-être un peu d'humour mais surtout de l'ironie. Je crois que, même si on fait son travail sérieusement, on doit être capable de rire de soi de temps en temps. J'ai voulu dégonfler un peu tous ces grands intellectuels qui se prennent pour le nombril du monde. C'est un nombril qui a souvent moins d'intérêt que d'autres que je connais! C'est un livre qui n'a pas très bien marché. J'aimerais bien que quelqu'un le réédite un jour. Peut-être qu'il aurait alors plus de succès. Car les universitaires sont moins gourmés aujourd'hui qu'il y a dix ou quinze ans.

D.S. Dans Un paien chez les pingouins, vous ridiculisez le milieu universitaire. Bessette fait la même chose dans Le Semestre, comme quoi ceux qui font la littérature ne se sentent pas tellement à l'aise parmi ceux qui la critiquent et qui en parlent dans leurs cours.

A.T. Je me sens à l'aise en classe, du moins je le crois. Je ne me suis jamais senti à l'aise parmi les intellectuels. Je déteste les réunions où l'on discute à grands renforts de mots savants et d'expressions choisies. Les créateurs, j'ai l'impression, ne vivent pas sur la même longueur d'onde que ceux qui décortiquent la création. Je me demande comment on fait pour concilier les modes de vie du créateur et du critique. Je le fais parce que ca me plaît mais je ne veux rien savoir de plus.

D.S. Vous avez publié une autre œuvre où le narrateur est un professeur. II s'agit de Soliloque en hommage à une femme (Cercle du livre de France, 1960). Je sais que vous pensez que Soliloque est un de vos meilleurs livres. Pourquoi? 
A.T. Pour toutes sortes de raisons. D'abord, je crois que ce livre est très bien écrit. Deuxièmement, les histoires des deux personnages principaux sont racontées par bribes, avec des retours en arrière mais à la fin, on finit par bien sentir leur passion. Enfin, il y a là un portrait du Québécois des années quarante qui, parti de très loin, a réussi, à force de volonté, à s'éduquer un peu et à commencer à vivre. Et ce personnage prend la peine de se définir, vers la fin du livre, avec des mots et des expressions qui me plaisent encore aujourd'hui.

D.S. Je vous avoue que la dimension poétique de Soliloque m'a tout à fait emballé. Vous avez écrit des passages splendides. Celui-ci, par exemple: "Je veux que tu me connaisses jusqu'en Saint-Hubert, jusqu'en mon pays, jusqu'en mes montagnes (...). J'appartiens à cette terre ingrate, même si à certains jours, je l'ai détestée, je l'ai honnie. J'appartiens à ce flanc de côteau pauvre, même si j'ai dû m'y laisser roussir au soleil et au vent au milieu des mauvaises herbes qui y poussaient mieux que l'orge et l'avoine (...). J'ai été attiré par les lointains et je suis parti. Mais je n'ai jamais pu arracher de ce sol les racines qui m'y retenaient. Elles ont continué de me nourrir, de $m^{\prime}$ apporter les sucs amers qui se sont mélangés aux sels de ce qu'on appelle la civilisation» (p. 19-21).

A.T. C'est un beau passage que vous citez là. Votre question me prouve que vous comprenez pourquoi j'aime beaucoup ce livre.

D.S. Mes Beaux Meurtres, recueil de cinq nouvelles publié au Cercle du livre de France en 1961 et réédité en 1973, est un livre qui a été reçu très favorablement. Les critiques ont aimé votre style ramassé. lls ont apprécié l'insolite et la fantaisie du recueil. C'est la violence qui m'a frappé personnellement. Dans la dernière nouvelle, vous expliquez pourquoi tant de personnages ont recours à la violence : $«(\ldots)$ je suis comme tout le monde, il m'arrive d'avoir des pensées que je me hâte d'enfouir au fond de ma conscience, d'avoir des désirs que j'aurais honte de montrer au grand jour, des sentiments que je rougirais parfois de dévoiler. D'être sujet à ces pensées, ces désirs, ces sentiments ne sert qu'à prouver que j'appartiens à l'espèce humaine» (p. 145).

A.T. La violence, elle est autour de nous partout. Comment en serait-it autrement alors qu'il y a tant d'injustices dans le monde? Cela rejoint un autre thème qui revient à plusieurs reprises dans mes livres: l'injustice. Tout le monde a envie de se révolter contre l'injustice. Peu de gens en ont les moyens. Les premiers grévistes qui se sont révoltés contre leurs patrons ont passé pour des perturbateurs de l'ordre social. Les puissants font tout pour accréditer l'idée que les pauvres sont des salauds, des gens sans conscience, des êtres immoraux, alors que les salauds, ce sont eux. II faut bien qu'ils jouent ce jeu s'ils veulent préserver leur puissance. Comment expliquer que si peu de gens voient clair dans ce jeu? Ou plutôt, que si peu de gens aient le courage de les dénoncer? Si on ne dénonce pas les salauds, en prenant tous les risques, qu'est-ce que le monde va devenir? 
D.S. Dans La Tête en fête, recueil d'«histoires étranges» qui s'apparentent aux nouvelles de Mes Beaux Meurtres, la folie est synonyme d'imagination. Dans C'est ici que le monde a commencé, vous écrivez: "C'est une bonne chose d'être fou de temps en temps. Ça ferait du bien à tout le monde."

A.T. Vous savez, j'avais d'abord intitulé La Tête en fête, La Folie en tête. Un mois ou deux avant la sortie du livre, j'ai vu dans une librairie un livre de Violette Leduc qui portait justement ce titre. J'ai dû trouver un autre titre. J'ai pris le titre d'une des histoires, intitulée La Tête en fête. Mais ce titre portait à faux puisqu'il donnait l'impression au lecteur, avant de commencer, qu'il s'agissait d'histoires drôlatiques. En fait, quand on y regarde de près, il s'agit d'histoires dramatiques où la folie prend une grande place.

On se retrouve tous, à certains moments de la vie, au bord de la folie et il faut faire quelquefois un effort énorme pour ne pas se faire avaler par elle. C'est ce que j'ai voulu dire, exprimer, dans La Tête en fête qui est justement l'histoire d'un garçon qui se retrouve à l'asile.

Quand je dis que ça ferait du bien à tout le monde d'être fou de temps en temps, je veux simplement dire que ça ferait du bien aux gens d'écouter les impulsions profondes qui remontent à la surface de leur conscience, mais qu'ils enfouissent tout de suite parce que la société dans laquelle on vit a placé des bornes partout. Instinctivement, la majorité des gens refusent de jouir à cause d'une fausse morale qu'ils acceptent comme la bonne morale. Quand on dit non à cette fausse morale, on passe pour fou. Les gens bien, les puissants, font tellement de saloperies à ceux qui n'ont pas les moyens de se défendre, en se couvrant du manteau de la bonne morale, que c'en est effrayant!

D.S. Vous avez publié deux pièces de théâtre. Dans ces œuvres, vous vous éloignez de votre Chemin Taché et de vos «histoires étranges». Comme dramaturge, c'est le Québec duplessiste (Les Renégats, Jumonville, 1964) et le phénomène des millionnaires anglo-saxons du Québec (Le Roi d'Aragon, Jumonville, 1979) qui vous intéressent. Pourquoi avoir introduit l'actualité dans ces pièces de théâtre, alors que dans vos romans et nouvelles, vous optez surtout pour le passé (votre enfance à SaintCyprien) ou pour le monde des pulsions intérieures?

A.T. J'ai écrit Les Renégats, dont le premier titre était Les Jouets mécaniques, en 1959. pendant que j'étais à l'Université Notre-Dame. J'en ai discuté avec le directeur de théâtre de I'Université qui aurait bien voulu pouvoir lire ma pièce en anglais. Mais je n'ai jamais pensé au régime duplessiste en écrivant ce texte. Les puissants comme ce père de famille riche - le personnage principal - qui obligent ceux qui les entourent à être leurs esclaves, ca existe depuis le commencement de l'humanité. Duplessis n'y est pour rien. 
Dans Le Roi d'Aragon, il sagit encore de puissants. Des gens qui détiennent le pouvoir sans qu'ils n'aient rien fait pour le mériter mais qui sont convaincus que la vérité leur appartient. Le grand thème de ces deux pièces, si on y regarde bien, c'est l'injustice des puissants à l'égard des démunis. Ce thème, je l'ai dit, se retrouve, un peu voilé peut-être, dans tous mes romans. L'histoire de Zénon qu'on appelle Zénon le souillon, dans la dernière partie de C'est ici que le monde a commencé, en est un bon exemple. C'est la petite société dans laquelle il vit qui prend la place des puissants, ici.

D.S. Dans Le Roi d'Aragon, des moines enlèvent un capitaliste canadienanglais. Les hommes d'Église vous fascinent. Vous aimez en faire des personnages. Dans Les Fous d'amour (Jumonville, 1973), tous les personnages sont des moines. Vous semblez admirer l'imagination, le mystère, la prestance et surtout la verve de certains hommes d'Église. Les prédicateurs, en qui les émotions existaient, en bons manichéens, à l'état pur et excessif, étaient en quelque sorte des personnages littéraires.

A.T. Tous les êtres qu'on rencontre dans la vie peuvent être des personnages littéraires. II suffit qu'ils nous inspirent. On peut alors les faire renaître en pleine fiction et, avec un peu de chance, leur souffler une nouvelle vie plus vraie que la premiere. Par ailleurs, les gens qui renoncent aux joies de la vie, pour celles d'une autre vie, m'ont toujours intrigué. Ils doivent se faire tellement violence, il me semble, pour continuer à vivre! Ce sont de grands refoulés. C'est un peu pour assister à leur défoulement que j'essaie de me mettre dans leur peau, de temps en temps. Je crois que je ne pourrais pas être moine sans que la déraison s'installe en moi.

D.S. Vous dites que vous moines sont des "fous d'amour". Mais ce sont plus des adeptes du marquis de Sade que des enfants de Marie.

A.T. Mais non! Mais non! Mes moines sont tous de bons moines qui ne songent même pas à remettre leur vocation en cause. Mais ce sont des êtres passionnés comme d'autres et, à certains moments, cette passion, à cause de certaines circonstances, ne peut faire autrement que d'éclater au grand jour. Pourquoi voudriez-vous que des moines ne soient pas harcelés par leurs passions? S'ils ne l'étaient pas, ils n'auraient aucun mérite à faire ce qu'ils font. On peut bien refouler ses désirs pendant un certain temps mais ils peuvent se montrer si forts qu'ils remonteront à la surface et c'est alors le drame. On ne cesse pas d'être un être humain parce qu'on devient moine. II $y$ a eu toutes sortes de drames dans les monastères au Moyen Âge, je suis sûr qu'il y en a encore aujourd'hui.

D.S. Adrien Thério, merci de nous avoir parlé de votre vie et de votre ceuvre. Je vous souhaite de bonnes écritures. Les gens du Chemin Taché, vos moines jouisseurs et tous vos êtres à l'imagination fertile vous attendent. Vos lecteurs aussi. 